\title{
Positive periodic solutions of functional discrete systems with a parameter
}

\author{
Youssef N. RAFFOUL \\ Department of Mathematics, \\ University of Dayton, Dayton, $\mathrm{OH}$ 45469-2316 \\ yraffoul1@udayton. edu \\ ERNEST YANKSON \\ Department of Mathematics and Statistics, \\ University of Cape Coast, Cape Coast, Ghana. \\ ernestoyank@gmail.com
}

\begin{abstract}
The existence of multiple positive periodic solutions of the system of difference equations with a parameter

$$
x(n+1)=A(n, x(n)) x(n)+\lambda f\left(n, x_{n}\right),
$$

is studied. In particular, we use the eigenvalue problems of completely continuous operators to obtain our results. We apply our results to a well-known model in population dynamics.
\end{abstract}




\section{RESUMEN}

Estudiamos la existencia de soluciones periódicas múltiples del siguiente sistema de ecuaciones diferenciales con un parámetro

$$
x(n+1)=A(n, x(n)) x(n)+\lambda f\left(n, x_{n}\right) .
$$

En particular, usamos los problemas de valores propios de operadores completamente continuos para obtener nuestros resultados. Aplicamos nuestros resultados a modelos de dinámica poblacional bien conocidos.

Keywords and Phrases: Functional difference system, Positive periodic solution, Eigenvalue, Population model

2010 AMS Mathematics Subject Classification: 39A10, 39A12. 


\section{Introduction}

Let $\mathbb{R}$ denote the real numbers, $\mathbb{Z}$ the integers, $\mathbb{Z}_{-}$the negative integers, $\mathbb{R}_{+}^{k}=\left\{\left(x_{1}, x_{2}, \ldots, x_{k}\right)^{\top} \in\right.$ $\left.\mathbb{R}^{k}: x_{j} \geq 0, j=1,2, \ldots, k\right\}, \mathbb{R}^{+}=\{x \in \mathbb{R}: x>0\}$, and $\mathbb{Z}^{+}$the nonnegative integers. Also, let BC denote the normed vector space of bounded functions $\phi: \mathbb{Z} \rightarrow \mathbb{R}^{k}$, with the norm $\|\phi\|=\sum_{j=1}^{k} \max _{n \in[0, \omega-1]}\left|\phi_{j}(n)\right|$, where $\phi=\left(\phi_{1}, \phi_{2}, \ldots, \phi_{k}\right)^{\top}$ and $[0, \omega-1]=\{0,1, \ldots, \omega-1\}$. Particularly for each $x=\left(x_{1}, x_{2}, \ldots, x_{k}\right)^{\top} \in \mathbb{R}^{k}$, we define the norm $|x|_{0}=\sum_{j=1}^{k}\left|x_{j}\right|$. Also, denote by $B C_{+}^{k}=\left\{\phi \in B C: \phi(n) \in \mathbb{R}_{+}^{k}\right.$ for $\left.n \in \mathbb{Z}\right\}$.

In 12, Raffoul used a Krasnoselskii's fixed point theorem in cones to prove the existence of positive periodic solutions of the scaler difference equation with parameter

$$
x(n+1)=a(n) x(n)+\lambda h(n) f(x(n-\tau(n))) .
$$

Also, in [10], Zhu and Li generalized the work in [12] by proving that the system of difference equations with parameter

$$
x(n+1)=A(n) x(n)+\lambda h(n) f(x(n-\tau(n)))
$$

where $A(n)=\operatorname{diag}\left[a_{1}(n), a_{2}(n), \ldots, a_{m}(n)\right]$ and $h(n)=\operatorname{diag}\left[h_{1}(n), h_{2}(n), \ldots, h_{m}(n)\right]$ has positive periodic solutions. Motivated by the above considerations we investigate the existence of multiple positive periodic solutions of the nonautonomous system of difference equations

$$
x(n+1)=A(n, x(n)) x(n)+\lambda f\left(n, x_{n}\right),
$$

where, $\lambda>0$ is a parameter, $A(n, x(n))=\operatorname{diag}\left[a_{1}(n, x(n)), \ldots, a_{k}(n, x(n))\right], a_{j}(n+w,)=.a_{j}(n,$.$) ,$ $f(n, x): \mathbb{Z} \times B C \rightarrow \mathbb{R}^{k}$ is continuous in $x$ and $f(n, x)$ is $\omega$-periodic in $n$ and $x$, whenever $x$ is $\omega$ periodic, $\omega \geq 1$ is an integer. If $x \in B C$, then $x_{n} \in B C$ for any $n \in \mathbb{Z}$ is defined by $x_{n}(\theta)=x(n+\theta)$ for $\theta \in \mathbb{Z}$. Throughout this paper, we denote the product of $y(n)$ from $n=a$ to $n=b$ by $\prod_{n=a}^{b} y(n)$ with the understanding that $\prod_{n=a}^{b} y(n)=1$ for all $a>b$. Also, for two $m \times n$ matrices $A$ and $B, A \geq B(A<B)$ means that the inequality is satisfied entrywisely. In particular, $A$ is said to be a nonnegative matrix if $A \geq 0$.

Definition 3.1. 4 Let $\mathrm{X}$ be a Banach space and $\mathrm{P}$ a closed, nonempty subset of $\mathrm{X}$. $\mathrm{P}$ is a (convex) cone if

(i) $x, y \in P$ and $\alpha, \beta \in \mathbb{R}_{+}$imply $\alpha x+\beta y \in P$.

(ii) $x \in P$ and $-x \in P$ imply $x=0$.

Definition 3.2. 4] Let $X$ be a Banach space and $D \subset X, 0 \in D$. The operator $L: D \rightarrow X$ is such that $L O=0 . x_{\lambda} \neq 0$ is said to be an eigenvector of the eigenvalue $\lambda$ of $L$ if $L x_{\lambda}=\lambda x_{\lambda}$. 
Lemma 3.1. 4 Suppose $D$ is an open subset of an infinite-dimensional real Banach space $X$, $0 \in \mathrm{D}$, and $\mathrm{P}$ is a cone of $\mathrm{X}$. If the operator $\Gamma: \mathrm{P} \cap \overline{\mathrm{D}} \rightarrow \mathrm{P}$ is completely continuous with $\Gamma 0=0$ and satisfies $\inf _{x \in P \cap \partial D}\|\Gamma x\|>0$, then $\Gamma$ has an eigenvector on $P \cap \partial D$ associated with a positive eigenvalue. That is, there exist $x_{0} \in P \cap \partial D$ and $\mu_{0}>0$ such that $\Gamma x_{0}=\mu_{0} x_{0}$.

In this paper we make the following assumptions.

(H1) $0<a_{j}(n)<1, j=1,2, \ldots k$, and $n \in[0, \omega-1]$.

$(H 2)$ There exist $B(n)=\operatorname{diag}\left[b_{1}(n), b_{2}(n), \ldots, b_{k}(n)\right]$ and $C(n)=\operatorname{diag}\left[c_{1}(n), c_{2}(n), \ldots, c_{k}(n)\right]$ where $b_{j}, c_{j}: \mathbb{Z} \rightarrow \mathbb{R}_{+}$are $\omega$-periodic with $0<b_{j}, c_{j}<1$, such that

$$
\mathrm{B}(\mathrm{n}) \leq \mathrm{A}(\mathrm{n}, \varphi(\mathrm{n})) \leq \mathrm{C}(\mathrm{n})
$$

for all $(n, \varphi) \in \mathbb{Z} \times B C_{+}^{k}$.

(H3) $f(n, 0)=0$ for all $n \in \mathbb{Z}$.

(H4) $\mathrm{f}\left(\mathrm{n}, \varphi_{\mathrm{n}}\right) \leq 0$ for all $(\mathrm{n}, \varphi) \in \mathbb{Z} \times \mathrm{BC}_{+}^{\mathrm{k}}$.

(H5) For any $\mathrm{L}>0$ and $\epsilon>0$, there exists $\delta>0$ such that $\left[\phi, \psi \in \mathrm{BC}_{+}^{k},\|\phi\| \leq \mathrm{L},\|\psi\| \leq\right.$ L, $\|\phi-\psi\|<\delta, 0 \leq s \leq \omega]$ imply

$$
\left|f\left(s, \phi_{s}\right)-f\left(s, \psi_{s}\right)\right|<\epsilon .
$$

To study system (1.1) we let $\mathcal{X}=\left\{x: \mathbb{Z} \rightarrow \mathbb{R}^{k}, x(n+w)=x(n)\right\}$, then it is clear that $\mathcal{X} \subset \mathrm{BC}$, endowed with the norm $\|x\|=\sum_{j=1}^{k}\left|x_{j}\right|_{0}$, where $\left|x_{j}\right|_{0}=\max _{n \in[0, \omega-1]}\left|x_{j}(n)\right|$.

For the next lemma we consider

$$
x_{j}(n+1)=a_{j}(n, x(n)) x_{j}(n)+f_{j}\left(n, x_{n}\right), j=1,2, \ldots, k .
$$

The proof of the next lemma can be easily deduced from 12 and hence we omit it.

Lemma 3.2. Suppose that (H1) hold. If $x(n) \in \mathcal{X}$ then $x_{\mathfrak{j}}(n)$ is a solution of equation (1.2) if and only if

$$
x_{j}(n)=\sum_{u=n}^{n+T-1} G_{j}^{x}(n, u) f_{j}\left(n, x_{n}\right), j=1,2, \ldots, k,
$$

where

$$
G_{j}^{x}(n, u)=\frac{\prod_{s=u+1}^{n+T-1} a_{j}(s, x(s))}{1-\prod_{s=n}^{n+T-1} a_{j}(s, x(s))}, \quad u \in[n, n+T-1], j=1,2, \ldots, k .
$$


Let

$$
\sigma=\min _{1 \leq j \leq k} \frac{\left(\prod_{s=0}^{w-1} b_{j}(s)\right)\left[1-\prod_{s=0}^{w-1} c_{j}(s)\right]}{\left(\prod_{s=0}^{w-1} c_{j}(s)\right)\left[1-\prod_{s=0}^{w-1} b_{j}(s)\right]}
$$

It can easily be obtained from (H2) that $\sigma<1$. We next define two cones in $\mathcal{X}$ as follows:

$$
P_{1}=\left\{y \in \mathcal{X}: y_{j}(n) \geq \sigma\left|y_{j}\right|_{0}, n \in \mathbb{Z} \quad \text { and } j=1, \ldots, k\right\}
$$

and

$$
P_{2}=\{y \in \mathcal{X}: y(n) \geq 0, n \in \mathbb{Z}\}
$$

Define an operator $\mathrm{T}$ on $\mathcal{X}$ by $\mathrm{T}: \mathcal{X} \rightarrow \mathcal{X}$ by

$$
(T x)=\left(T_{1} x, T_{2} x, \ldots, T_{k} x\right)^{\top}
$$

where

$$
\left(T_{j} x\right)(n)=\sum_{u=n}^{n+w-1} G_{j}^{x}(n, u) f_{j}\left(u, x_{u}\right), j=1, \ldots, k .
$$

It is not very difficult to see that $G_{j}^{x}(n+w, u+w)=G_{j}^{x}(n, u)$. Also, it can easily be verified that $\chi^{*}(n)=\left(x_{1}^{*}(n), \ldots, x_{k}^{*}(n)\right) \geq 0$ is a positive $\omega$-periodic solution of system (1.1) associated with $\lambda^{*}$ if and only if $x^{*} \in P_{2}$ is an eigenvector of the operator $T$ associated with the eigenvalue $\frac{1}{\lambda^{*}}>0$, that is $T x^{*}=\frac{1}{\lambda^{*}} x^{*}$.

Lemma 3.2. Suppose that $(H 1)$ and $(H 2)$ hold. Then the mapping $T$ maps $P_{1}$ into $P_{1}$, i.e., $\mathrm{TP}_{1} \subset \mathrm{P}_{1}$.

Proof. In view of (H1) and (H2), we have that, for $j=1,2, \ldots, k$, and $0 \leq u \leq w-1$,

$$
\frac{\prod_{s=0}^{\omega-1} b_{j}(s)}{1-\prod_{s=0}^{\omega-1} b_{j}(s)} \leq G_{j}^{x}(n, u) \leq \frac{\prod_{s=0}^{\omega-1} c_{j}(s)}{1-\prod_{s=0}^{\omega-1} c_{j}(s)}
$$




$$
\begin{aligned}
\left|\left(T_{j} x\right)(n)\right| & \leq \sum_{u=n}^{n+w-1} \frac{\prod_{s=0}^{w-1} c_{j}(s)}{1-\prod_{s=0}^{w-1} c_{j}(s)}\left|f_{j}\left(u, x_{u}\right)\right| \\
& \leq \frac{\prod_{s=0}^{w-1} c_{j}(s)}{1-\prod_{s=0}^{w-1} c_{j}(s)} \sum_{u=0}^{\omega-1}\left|f_{j}\left(u, x_{u}\right)\right|
\end{aligned}
$$

It follows that

$$
\left|\left(T_{j} x\right)\right|_{0} \leq \frac{\prod_{s=0}^{w-1} c_{j}(s)}{1-\prod_{s=0}^{w-1} c_{j}(s)} \sum_{u=0}^{\omega-1}\left|f_{j}\left(u, x_{u}\right)\right|
$$

or

$$
\sum_{u=0}^{\omega-1}\left|f_{j}\left(u, x_{u}\right)\right| \geq \frac{1-\prod_{s=0}^{w-1} c_{j}(s)}{\prod_{s=0}^{\omega-1} c_{j}(s)}\left|\left(T_{j} x\right)\right|_{0} .
$$

Therefore,

$$
\begin{aligned}
\left(T_{j} x\right)(n) & \geq \frac{\prod_{s=0}^{\omega-1} b_{j}(s)}{1-\prod_{s=0}^{\omega-1} b_{j}(s)} \sum_{u=0}^{\omega-1}\left|f_{j}\left(u, x_{\mathfrak{u}}\right)\right| \\
& \geq \frac{\left(\prod_{s=0}^{\omega-1} b_{j}(s)\right)\left[1-\prod_{s=0}^{\omega-1} c_{j}(s)\right]}{\left(\prod_{s=0}^{\omega-1} c_{j}(s)\right)\left[1-\prod_{s=0}^{\omega-1} b_{j}(s)\right]}\left|\left(T_{j} x\right)\right|_{o} \\
& \geq \sigma\left|\left(T_{j} x\right)\right|_{o},
\end{aligned}
$$

which means that $T x \in \mathrm{P}_{1}$. This completes the proof.

Lemma 3.3. Suppose (H5) hold. Then the operator $\mathrm{T}: \mathrm{P}_{2} \rightarrow \mathcal{X}$ is completely continuous.

Proof. In view of (H5) and the assumption that $f(n, x)$ is continuous in $x$, we have that the operator $T$ is continuous. We will show that $T$ is compact.

Let $\mathrm{U} \subseteq \mathrm{P}_{2}$ be any bounded set. Then, by the (H5), there exists a constant $\mathrm{M}>0$ such that

$$
\left|f_{j}\left(n, x_{n}\right)\right| \leq M, \text { for }(n, x) \in[0, \omega-1] \times U, j=1,2, \ldots, k .
$$

Thus we have,

$$
\left|\left(T_{j} x\right)\right| \leq \frac{\prod_{s=0}^{\omega-1} c_{j}(s)}{1-\prod_{s=0}^{w-1} c_{j}(s)} M \omega
$$

It follows that, 


$$
\begin{aligned}
\|(T x)\| & =\sum_{j=1}^{k}\left|T_{j} x\right|_{0} \\
& \leq M \omega \sum_{j=1}^{k} \frac{\prod_{s=0}^{\omega-1} c_{j}(s)}{1-\prod_{s=0}^{\omega-1} c_{j}(s)} \\
& \leq M k \omega \gamma,
\end{aligned}
$$

where

$$
\gamma=\max _{1 \leq j \leq k} \frac{\prod_{s=0}^{\omega-1} c_{j}(s)}{1-\prod_{s=0}^{\omega-1} c_{j}(s)} .
$$

Next, we show that $T$ maps bounded subsets into compact sets. Let $\mathrm{J}>0$ be given, and define $\rho=\left\{\varphi \in \mathrm{P}_{2}:\|\varphi\| \leq J\right\}$ and $\mathrm{Q}=\{(\mathrm{T} \varphi)(\mathrm{n}): \varphi \in \rho\}$, then $\rho$ is a subset of $\mathbb{R}^{\omega k}$ which is closed and bounded thus compact. As $T$ is continuous in $\varphi$ it maps compact sets into compact sets. Therefore $\mathrm{Q}=\mathrm{T}(\rho)$ is compact.

This completes the proof of lemma 3.3.

\section{Main Results}

In this section we state and prove our main results. For our main results we let

$$
f_{0}=\lim _{\phi \in P_{1},\|\phi\| \rightarrow 0} \frac{\sum_{u=0}^{\omega-1}\left|f\left(u, x_{\mathfrak{u}}\right)\right|}{\|\phi\|} \text {, and } f_{\infty}=\lim _{\phi \in P_{1},\|\phi\| \rightarrow \infty} \frac{\sum_{u=0}^{\omega-1}\left|f\left(u, x_{\mathfrak{u}}\right)\right|}{\|\phi\|} .
$$

Also, define, for $r$ a positive number, $\Omega_{r}$, by

$$
\Omega_{r}=\{x \in \mathcal{X}:\|x\|<r\} .
$$

Theorem 4.1 Suppose that (H1)-(H5) hold and $0<\mathrm{f}_{\infty}<\infty$. Then there exist positive constants $R_{0}, \lambda_{1}$, and $\lambda_{2}$ with $\lambda_{1}<\lambda_{2}$ such that, for any $r>R_{0}$, system (1.1) has a positive $\omega$-periodic solution $x^{r}(n)$ associated with some $\lambda_{r} \in\left[\lambda_{1}, \lambda_{2}\right]$ and $\left\|x^{r}\right\|=r$.

Proof. Since $0<f_{\infty}<+\infty$, there exist $\epsilon_{2}>\epsilon_{1}>0$ and $R_{0}>0$ such that

$$
\epsilon_{1}\|\phi\|<\sum_{\mathfrak{u}=0}^{\omega-1}\left|f\left(u, \phi_{u}\right)\right|<\epsilon_{2}\|\phi\| \text { for }\|\phi\| \geq R_{0}, \phi \in P_{1} .
$$


Suppose $r>R_{0}$, then $\Omega_{r}$ is a bounded open subset of $\mathcal{X}$ and $0 \in \Omega_{r}$. For $x \in P_{1} \cap \partial \Omega_{r}$, we have

$$
\begin{aligned}
\|T x\| & =\sum_{j=1}^{k} \max _{n \in[0, \omega-1]}\left|\left(T_{j} x\right)(n)\right| \\
& \geq \sum_{j=1}^{k}\left|\left(T_{j} x\right)(n)\right| \\
& =\sum_{j=1}^{k} \sum_{u=0}^{\omega-1} G_{j}^{x}(n, u) f_{j}\left(u, x_{\mathfrak{u}}\right) \\
& \geq \sum_{j=1}^{k} \frac{\prod_{s=0}^{\omega-1} b_{j}(s)}{1-\prod_{s=0}^{\omega-1} b_{j}(s)} \sum_{u=0}^{\omega-1} f_{j}\left(u, x_{\mathfrak{u}}\right) \\
& \geq \min _{1 \leq j \leq k} \frac{\prod_{s=0}^{\omega-1} b_{j}(s)}{1-\prod_{s=0}^{\omega-1} b_{j}(s)} \sum_{u=0}^{\omega-1} \sum_{j=1}^{k}\left|f_{j}\left(u, x_{\mathfrak{u}}\right)\right| \\
& \geq \min _{1 \leq j \leq k} \frac{\prod_{s=0}^{\omega-1} b_{j}(s)}{1-\prod_{s=0}^{\omega-1} b_{j}(s)} \epsilon_{1} r>0 .
\end{aligned}
$$

It follows that

$$
\inf _{x \in P_{1} \cap \partial \Omega_{r}}\|T x\| \geq \min _{1 \leq j \leq k}\left\{\frac{\prod_{s=0}^{w-1} b_{j}(s)}{1-\prod_{s=0}^{\omega-1} b_{j}(s)}\right\} \epsilon_{1} r>0 .
$$

Since, $\mathrm{T}$ is completely continuous with $\mathrm{T}(0)=0$, it follows from Lemma 3.1 that the operator $\mathrm{T}$ has an eigenvector $x^{r} \in P_{1}$ associated with the eigenvalue $\mu_{r}>0$ such that $\left\|x^{r}\right\|=r$. Set $\lambda_{r}=\frac{1}{\mu_{r}}$. Then $x^{r}$ is a positive $\omega$-periodic solution of system (1.1).

We next determine $\lambda_{1}$ and $\lambda_{2}$ as follows. From

$$
\begin{aligned}
\left(x^{r}\right)_{j}(n) & =\lambda_{r} \sum_{u=n}^{n+\omega-1} G_{j}^{x^{r}}(n, u) f_{j}\left(u, x_{u}^{r}\right) \\
& \leq \lambda_{r} \sum_{u=0}^{\omega-1} \frac{\prod_{s=0}^{\omega-1} c_{j}(s)}{1-\prod_{s=0}^{\omega-1} c_{j}(s)}\left|f_{j}\left(u, x_{u}^{r}\right)\right| \\
& \leq \lambda_{r} \frac{\prod_{s=0}^{w-1} c_{j}(s)}{1-\prod_{s=0}^{\omega-1} c_{j}(s)} \sum_{u=0}^{\omega-1}\left|f_{j}\left(u, x_{u}^{r}\right)\right| \\
& \leq \lambda_{r} \frac{\prod_{s=0}^{\omega-1} c_{j}(s)}{1-\prod_{s=0}^{w-1} c_{j}(s)} \epsilon_{2} r, j=1,2, \ldots, k,
\end{aligned}
$$

and $\left\|x^{r}\right\|=r$ we can get

$$
\lambda_{r} \geq \frac{1}{\epsilon_{2} \sum_{j=1}^{k} \frac{\prod_{s=0}^{\omega-1} c_{j}(s)}{1-\prod_{s=0}^{\omega-1} c_{j}(s)}}=: \lambda_{1}
$$


On the other hand,

$$
\left(x^{r}\right)_{j}(n) \geq \lambda_{r} \frac{\prod_{s=0}^{w-1} b_{j}(s)}{1-\prod_{s=0}^{\omega-1} b_{j}(s)} \sum_{u=0}^{\omega-1}\left|f_{j}\left(u, x_{\mathfrak{u}}^{r}\right)\right|, j=1, \ldots, k .
$$

It follows from

$$
\begin{aligned}
\left\|x^{r}\right\|=r & \geq \lambda_{r} \min _{1 \leq j \leq k}\left\{\frac{\prod_{s=0}^{\omega-1} b_{j}(s)}{1-\prod_{s=0}^{\omega-1} b_{j}(s)}\right\} \sum_{u=0}^{\omega-1}\left|f\left(u, x_{u}^{r}\right)\right| \\
& \geq \lambda_{r} \min _{1 \leq j \leq k}\left\{\frac{\prod_{s=0}^{\omega-1} b_{j}(s)}{1-\prod_{s=0}^{\omega-1} b_{j}(s)}\right\} \epsilon_{1} r
\end{aligned}
$$

that

$$
\lambda_{r} \leq \lambda_{r} \max _{1 \leq j \leq k}\left\{\frac{1-\prod_{s=0}^{\omega-1} b_{j}(s)}{\epsilon_{1} \prod_{s=0}^{\omega-1} b_{j}(s)}\right\}:=\lambda_{2}
$$

Therefore, $\lambda_{\mathrm{r}} \in\left[\lambda_{1}, \lambda_{2}\right]$ and this completes the proof.

Theorem 4.2. Suppose that (H1)-(H5) hold and $0<f_{0}<\infty$. Then there exist positive constants $r_{0}>0, \tilde{\lambda_{1}}$ and $\tilde{\lambda_{2}}$ with $\tilde{\lambda_{1}}<\tilde{\lambda_{2}}$ such that, for any $0<r<r_{0}$, system (1.1) has a positive $\omega$-periodic solution $\tilde{x^{r}}(n)$ associated with some $\tilde{\lambda_{r}} \in\left[\tilde{\lambda_{1}}, \tilde{\lambda_{2}}\right]$ and $\left\|\tilde{x^{r}}\right\|=r$.

Proof. Since $0<f_{0}<\infty$, there exist $0<l_{1}<l_{2}$ and $r_{0}>0$ such that

$$
\mathrm{l}_{1}\|\phi\|<\sum_{\mathfrak{u}=0}^{\omega-1}\left|\mathrm{f}\left(\mathrm{u}, \phi_{\mathfrak{u}}\right)\right|<\mathrm{l}_{2}\|\phi\| \text { for } 0<\|\phi\|<\mathrm{r}_{0}, \phi \in \mathrm{P}_{1} .
$$

For $r \in\left(0, r_{0}\right), \Omega_{r}$ is a bounded subset of $\mathcal{X}$ and $0 \in \Omega_{r}$. Moreover, for $x \in P_{1} \cap \partial \Omega_{r}$,

$$
\begin{aligned}
\|T x\| & \geq \sum_{j=1}^{k}\left|\left(T_{j} x\right)(n)\right| \\
& =\sum_{j=1}^{k} \sum_{u=n}^{n+w-1} G_{j}^{x}(n, u) f_{j}\left(u, x_{u}\right) \\
& \geq \min _{1 \leq j \leq k}\left\{\frac{\prod_{s=0}^{w-1} b_{j}(s)}{1-\prod_{s=0}^{w-1} b_{j}(s)}\right\} l_{1} r>0 .
\end{aligned}
$$

This implies that $\inf _{x \in \mathrm{P}_{1} \cap \partial \omega_{r}}\|\mathrm{Tx}\|>0$. The remaining part of the proof is similar to that of Theorem 4.1 and so we omit it. This completes the proof. 
Using arguments similar to that of Theorem 4.1 and Theorem 4.2, the following results can be established respectively.

Theorem 4.3. Suppose that (H1)-(H5) hold and $f_{\infty}=\infty$. Then there exist positive constants $\breve{R}_{0}$ and $\breve{\lambda}$ such that, for any $r>\breve{R_{0}}$, system (1.1) has a positive $\omega$-periodic solution $\breve{x}^{r}(n)$ associated with some $\breve{\lambda}_{r} \leq \breve{\lambda}$ and $\left\|\breve{x}^{r}\right\|=r$.

Theorem 4.4. Suppose that (H1)-(H5) hold and $f_{0}=\infty$. Then there exist positive constants $\bar{r}_{0}$ and $\bar{\lambda}$ such that, for any $0<r<\overline{r_{0}}$, system (1.1) has a positive $\omega$-periodic solution $\bar{\chi}^{r}(n)$ associated with some $\bar{\lambda}_{\mathrm{r}} \leq \bar{\lambda}$ and $\left\|\bar{\chi}^{\mathrm{r}}\right\|=\mathrm{r}$.

\section{An application}

In this section, we apply our results from the previous section to the Volterra discrete system

$$
\begin{gathered}
x_{j}(n+1)=x_{j}(n)\left[a_{j}(n)-\lambda \sum_{i=1}^{k}\left(b_{j i}(n) x_{i}(n)+\sum_{s=-\infty}^{n} C_{j i}(n, s) g_{j i}\left(x_{i}(s)\right)\right)\right], \\
j=1,2, \ldots, k
\end{gathered}
$$

where $x_{j}(n)$ is the population of the $j$ th species, $a_{j}, b_{j i}: \mathbb{Z} \rightarrow \mathbb{R}_{+}$are $\omega$-periodic and $C_{j i}(n, s) \geq 0$ and $C_{j i}(n+\omega, s+\omega)=C_{j i}(n, s)$ for all $(n, s) \in \mathbb{Z}^{2} ; g_{j i}: \mathbb{R}_{+} \rightarrow \mathbb{R}_{+}, i, j=1, \ldots, k$.

Theorem 5.1. Suppose that $\max _{n \in \mathbb{Z}} \sum_{s=-\infty}^{n}\left|C_{j i}(n, s)\right|<+\infty$. Then there exist positive constants $R_{0}$ and $\lambda_{0}$ such that, for any $r>R_{0}$, system (3.1) has a positive $\omega$-periodic solution $x^{r}(n)$ associated with $\lambda_{r} \leq \lambda_{0}$ and $\left\|x^{r}\right\|=r$.

Proof. Note that $A(n, x(n))=\operatorname{diag}\left[a_{1}(n), a_{2}(n), \ldots, a_{k}(n)\right]$ and $f=\left(f_{1}, f_{2}, \ldots, f_{k}\right)$ where

$$
f_{j}\left(n, x_{n}\right)=-x_{j}(n) \sum_{i=1}^{k}\left(b_{j i}(n) x_{i}(n)+\sum_{s=-\infty}^{n} C_{j i}(n, s) g_{j i}\left(x_{i}(s)\right)\right)
$$

for $j=1,2, \ldots, k$ and (H1)-(H5) are satisfied. 
For $x \in P_{1}$ and $j=1, \ldots, k$ we have

$$
\begin{aligned}
\sum_{u=0}^{\omega-1}\left|f_{j}\left(u, x_{u}\right)\right| & =\sum_{i=1}^{k} \sum_{u=o}^{\omega-1} x_{j}(u)\left(x_{i}(u) b_{j i}(u)+\sum_{s=-\infty}^{u} C_{j i}(u, s) g_{j i}\left(x_{i}(s)\right)\right) \\
& \geq \sum_{i=1}^{k} \sum_{u=o}^{\omega-1} x_{j}(u) x_{i}(u) b_{j i}(u) \\
& \geq \sum_{u=0}^{\omega-1} x_{j}^{2}(u) b_{j j}(u) \\
& \geq \sigma^{2}\left|x_{j}\right|_{0}^{2} \sum_{u=o}^{\omega-1} b_{j j}(u) .
\end{aligned}
$$

Thus,

$$
\begin{aligned}
\sum_{u=0}^{\omega-1}\left|f\left(u, x_{\mathfrak{u}}\right)\right| & =\sum_{j=1}^{k} \sum_{u=0}^{\omega-1}\left|f_{j}\left(u, x_{\mathfrak{u}}\right)\right| \\
& \geq \sum_{j=1}^{k} \sigma^{2}\left|x_{j}\right|_{0}^{2} \sum_{u=0}^{\omega-1} b_{j j}(u) \\
& \geq \sigma^{2} \min _{1 \leq j \leq k} \sum_{u=0}^{\omega-1} b_{j j}(u) \sum_{j=1}^{k}\left|x_{j}\right|_{0}^{2} \\
& \geq \frac{\sigma^{2}}{k}\|x\|^{2} \min _{1 \leq j \leq k} \sum_{u=0}^{\omega-1} b_{j j}(u) .
\end{aligned}
$$

It follows that

$$
\frac{\sum_{\mathfrak{u}=0}^{\omega-1}\left|\mathrm{f}\left(\mathrm{u}, \mathrm{x}_{\mathfrak{u}}\right)\right|}{\|\mathrm{x}\|} \rightarrow \text { as }\|\mathrm{x}\| \rightarrow \infty
$$

The conclusion follows directly from Theorem 4.3 and this completes the proof. 


\section{References}

[1] A. Datta and J. Henderson, Differences and smoothness of solutions for functional difference equations, Proceedings Difference Equations, 1 (1995), 133-142.

[2] Y. Chen, B. Dai and N. Zhang, Positive periodic solutions of non-autonomous functional differential systems, J. Math. Anal. Appl. 333 (2007) 667-678.

[3] S. N. Elaydi, An Introduction to Difference Equations, 2nd ed., Undergraduate Texts in Mathematics, Springer-Verlag, New York, 1999.

[4] D.J. Guo and V. Lakshmikantham, Nonlinear Problems in Abstract Cones, Notes and Reports in Mathe matics and Science and Engineering, vol. 5, Academic Press Inc., Boston, MA, 1988, pp. 2-99.

[5] J. Henderson and A. Peterson, Properties of delay variation in solutions of delay difference equations, Journal of Differential Equations, 1 (1995), 29-38.

[6] R.P. Agarwal and P.J.Y. Wong, On the existence of positive solutions of higher order difference equations, Topological Methods in Nonlinear Analysis, 10 (1997) 2, 339-351.

[7] P.W. Eloe, Y. Raffoul, D. Reid and K. Yin, Positive solutions of nonlinear Functional Difference Equations, Computers and Mathematics With applications, 42 (2001) , 639-646.

[8] J. Henderson and W. N. Hudson, Eigenvalue problems for nonlinear differential equations, Communications on Applied Nonlinear Analysis, 3 (1996), 51-58.

[9] M. A. Krasnosel'skii, Positive solutions of operator Equations, Noordhoff, Groningen, (1964).

[10] Y. Li and L. Zhu, Positive periodic solutions of higher-dimensional functional difference equations with a parameter, J. Math. Anal. Appl. 290 (2004) 654-664.

[11] F. Merdivenci, Two positive solutions of a boundary value problem for difference equations, Journal of Difference Equations and Application, 1 (1995), 263-270.

[12] Y.N. Raffoul, Positive periodic solutions of nonlinear functional difference equations, Electron. J. Differential Equations, 55 (2002) 1-8.

[13] Y.N. Raffoul, Periodic solutions for scalar and vector nonlinear difference equations, PanAmerican Journal of Mathematics, 9 (1999), 97-111.

[14] W. Yin, Eigenvalue problems for functional differential equations, Journal of Nonlinear Differential Equations, 3 (1997), 74-82. 